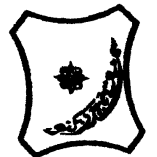

Bayero Journal of Pure and Applied Sciences, 5(1): 113 - 122

Received: November 2011

Accepted: May 2012

ISSN $2006-6996$

\title{
YIELD AND YIELD COMPONEMTS OF EXTRA EARLY MAIZE (ZEA MAYS L.) AS INFLUENCED BY INTRA-ROW SPACING, NITROGEN AND POULTRY MANURE RATES
}

\author{
Sharifai, A.I., Mahmud, M., Tanimu, B. and Abubakar, I.U. \\ Department of Agronomy Institute of Agricultiral Research, Ahmadu Bello University, Zaria \\ Correspondence author: aminusharifai@yahoo.co.uk, aminusharifai@gmail.com
}

\begin{abstract}
Field trials were conducted during the rainy seasons of 2006, 2007 and 2008 at the Institute for Agricultural Research (I.A.R.) Farm, Samaru to determine the performance of extra early maize (Zea mays L.) as affected by intra-row spacing, nitrogen and poultry manure rates. The treatments consisted of factorial combinations of three intra-row spacing (20, 25 and $30 \mathrm{~cm})$, three rates of nitrogen (40, 80 and $\left.120 \mathrm{~kg} \mathrm{ha}^{-1}\right)$ and four rates of poultry manure $\left(0,2,4\right.$ and $\left.6 \mathrm{t} \mathrm{ha} \mathrm{h}^{-1}\right)$. The treatments were laid out in a split-plot design with three replications. Combinations of nitrogen and poultry manure rates were assigned to main plot, while intra-row spacing was assigned to the sub plot. The results showed increase in intra-row spacing from 20 to $25 \mathrm{~cm}$ significantly increased number of rows $\mathrm{cob}^{-1}$, cob diameter, 100-grain weight and grain yield. The effect of nitrogen on such yield components as cob diameter, cob length and 100-grain weight was significant as the response was in the range of 80 to $120 \mathrm{~kg} \mathrm{ha}^{-1}$. Varying level of poultry manure had a significant effect on the performance of the crop. The high level of $2 t \mathrm{ta}^{-1}$ poultry manure significantly out yielded the control but at par with 4 and $6 \mathrm{t} \mathrm{ha} \mathrm{a}^{-1}$ in most of the yield attributes. The optimum yield (2.26 $\mathrm{tha}^{-1}$ ) was obtained by the combination of $25 \mathrm{~cm}$ intra row spacing, $82 \mathrm{~kg} \mathrm{~N} \mathrm{ha}^{-1}$ and $1.91 \mathrm{t}$ poultry manure ha-1 and should therefore be adopted by extra early maize farmers in Northern Guinea Savanna agro ecology.

Keywords: Nitrogen, intra-row spacing, poultry manure, cob diameter, cob length, 100-grain weight and grain yield.
\end{abstract}

\section{INTRODUCTION}

Maize (Zea mays L.) is one of the most important staple food crops in West and Central Africa. The Savanna of West and Central Africa has one of the greatest potential for its major production because of relatively higher incident of solar radiation and lower incident of pest and diseases during the cropping season (Badu-Apraku et al., 2006). In 2008, the world production was 822.7 million tonnes, 53.4 million tonnes for Africa and 7.5 million tonnes for Nigeria (FAO, 2010). Maize production has expanded dramatically in the Northern Guinea Savanna of West Africa where it has replaced traditional cereals and serves as both a food and a cash crop. In West Africa, Manyong et al. (1996) assessed maize as one of the five main crops of the farming systems in 124.7 million hectare or $72 \%$ of West Africa. The Northern Guinea Savanna alone took about $92 \%$ of total area grown to maize in Nigeria. Maize is also widely believed to have the greatest potential among food crops for attaining the technological breakthroughs that will improve food production in the region (Kamara and Sanginga, 2001).

Growing maize at appropriate spacing is one of the bases for higher yield, whereas intra-row spacing at sub optimum is a major constrain to attaining the yield potential of the crop (Alofe et al., 1988). Intrarow spacing for maximum grain yield in maize varies from 20 to $45 \mathrm{~cm}$ (Olson and Sanders, 1988). There is no single recommendation for all environments and all maize types and varieties because optimum spacing for optimum maize yield could vary depending on climatic factors such factors as soil fertility, variety and type, planting date and planting pattern among others (Luis, 2001). The intra-row spacing used by the local farmers for open pollinated extra-early maize was found to be the same as for hybrid, medium and late maturing varieties. This could be a reason for the low yield obtained by farmers. Morphologically, extra-early maize varieties are generally shorter in height (185$190 \mathrm{~cm}$ ), have fewer number of leaves per plant, flowering occurs at about 40 days after sowing (Elemo, 1997).

Because of the high nutrient demand by maize, its production requires high inputs of fertilizer. However, because of high cost, unavailability and low levels of soil organic matter, alternative organic sources of nutrients particularly $\mathrm{N}$ needs to be included in maize fertilization. The use of animal manure is needed to ensure an efficient nutrient management in the maizebased cropping systems in the Northern Guinea Savanna. Research conducted in Northern Guinea Savanna and elsewhere had shown great improvement in the yield of crop as a result of improvement in organic matter content of the soil (Boateng et al., 2006). The present study was therefore designed to determine the influence of intrarow spacing, nitrogen and poultry manure rates on the yield and yield components of extra-early maize. 


\section{MATERIALS AND METHODS}

Field trials were conducted during the rainy seasons of 2006, 2007 and 2008 at the experimental Farm of Institute for Agricultural Research (I.A.R.), Samaru $\left(11^{\circ} 11^{\prime} \mathrm{N} 7^{\circ} 38^{\prime} \mathrm{E}\right.$ and $686 \mathrm{~m}$ above sea level) located in the Northern Guinea savannah agro-ecological zone of Nigeria, to study the performance of extra-early maize (Zea mays L.) as affected by intra-row spacing, nitrogen and poultry manure rates. The treatments consisted of factorial combinations of three levels each of intra-row spacing $(20,25$ and $30 \mathrm{~cm})$, and nitrogen $\left(40,80\right.$ and $\left.120 \mathrm{~kg} \mathrm{ha}^{-1}\right)$ and four levels of poultry manure $\left(0,2,4\right.$ and $\left.6 \mathrm{t} \mathrm{ha}^{-1}\right)$. These treatments were laid out in a split plot design with combination of nitrogen and poultry manure rates assigned to the main plot, while intra-row spacing was assigned to the subplot and replicated three times. The gross plot size was $4.0 \mathrm{~m} \times 4.5 \mathrm{~m}\left(18 \mathrm{~m}^{2}\right)$ and net plot size was $4 \mathrm{~m} \times 1.5 \mathrm{~m}\left(6 \mathrm{~m}^{2}\right)$. Composite soil samples of the experimental site and poultry manure used during the experimental periods were collected prior to establishment of the trial for each year and analysed for their chemical properties in each year. The soil in the experimental area is sandy loam; low in organic carbon and total $\mathrm{N}$. While poultry manure used has high total N (Table 1), while details of meteorological records for the three years are presented in appendix I. An extra early maturing maize (Zea mays L.) variety SAMMAZ 12 (previously known as 95-TZEE-W) was used in the study. It was obtained from IAR seed Unit in Samaru, Zaria. The variety is suited to the Sudan and lowland ecologies because of its earliness (80-85 days). The grain is white, semi-dent and has yield potential of $2.0-2.8 \mathrm{t} \mathrm{ha}^{-1}$. It is resistant to maize streak virus and has a tendency of escaping drought because of its earliness.

Prior to land preparation, Glyphosate was applied at 4 litre ha $^{-1}$ in the experimental site. After 12 days of application the site was ploughed and harrowed to obtain a fine tilth in each season. Ridging was done at $75 \mathrm{~cm}$ apart and the field sub divided into plots and replications as per the experimental layout. Seeds were sown manually on 21 June, 12 July and 26 June of 2006, 2007 and 2008 respectively. Two seeds were planted per hole as per the intra-row spacing of 20, 25 and $30 \mathrm{~cm}$ respectively, keeping the inter row spacing of $75 \mathrm{~cm}$ constant. Seedlings were thinned to one plant per stand at two weeks after sowing (WAS), giving the population of 66 666, 53 333 and 44444 plants ha ${ }^{-1}$ respectively. Weeds were controlled with pre emergence herbicide Atrazine + Metalacholor (Primextra) at the rate of $2 \mathrm{~kg}$ a.i ha ${ }^{-1}$ applied one day after sowing. Remolding was done at 5 WAS to check the growth of emerging weeds. A basal fertilizer application of $19.97 \mathrm{~kg} \mathrm{~K} \mathrm{ha}^{-1}$ (45 kg $\left.\mathrm{K}_{2} \mathrm{O}\right)$ and $37.35 \mathrm{~kg} \mathrm{P} \mathrm{ha}^{-1}\left(45 \mathrm{~kg} \mathrm{P}_{2} \mathrm{O}_{5}\right)$ were applied at planting using muriate of potash (MOP) and single super phosphate (SSP) as sources respectively. The incorporation of poultry manure as per treatment was done two weeks before sowing. Nitrogen in the form of urea $(46 \% \mathrm{~N})$ was applied in two split doses as per treatment at 3 and 5 WAS by side dressing. There was no serious incidence of pest and disease recorded during the three cropping seasons. Therefore no control measure was taken. Harvesting was done when the crops reached physiological maturity i.e. when the attachment of the grain to the cob was observed to be black and leaves turned yellow and brown and kernels were dried in the field. The cobs were plucked, dehusked and further sun-dried. The cobs were then weighed, threshed and winnowed to obtain clean grain.

Data collected includes number of cobs plant ${ }^{-}$ 1 , number of kernel row $\mathrm{cob}^{-1}$, cob diameter, cob length, 100-grain weight and grain yield. All data collected were subjected to General Linear model procedure (GLM) of the Statistical Analysis System (SAS institute Inc. 1990) and differences between the treatments were compared using Duncan Multiple Range Test as described by Duncan (1955). Polynomial responses of grain yield to the treatments were done using a regression analysis as suggested by Barr and Goodnight (1972). Optimum levels of nitrogen and poultry manure for maximum grain yield were calculated as suggested by Garg and Bansal (1972) and Reddy et al. (1975).

\section{RESULTS}

Table 2 shows the effect of intra-row spacing, nitrogen and poultry manure rates on number of cob plant $^{-1}$, number of rows $\operatorname{cob}^{-1}$ and cob diameter. In all the years, there were no significant differences in all the intra-row spacing treatments on number of cob plant $^{-1}$. Highest intra-row spacing $(30 \mathrm{~cm})$ produced significantly more number of rows cob ${ }^{-1}$ in 2006, 2007 and combined seasons only than at $20 \mathrm{~cm}$. No significant difference among nitrogen treatments were observed on number of cob plant ${ }^{-1}$ and number of rows $\mathrm{cob}^{-1}$. In 2006 and 2007, increase in nitrogen from 40 to $80 \mathrm{~kg} \mathrm{ha}^{-1}$ produced significantly wider cobs. Further increase to $120 \mathrm{~kg} \mathrm{~N} \mathrm{ha}^{-1}$ had statistically similar effect. However in 2008 and combined seasons $80 \mathrm{~kg} \mathrm{~N}^{-1}$ produced significantly wider cobs only than at $40 \mathrm{~kg} \mathrm{~N} \mathrm{ha}^{-1}$. In all the parameters tested, poultry manure treatments had no significant effect in all the years except in combined seasons for number of cob plant ${ }^{-1}$, where $4 \mathrm{t}$ poultry manure ha ${ }^{-1}$ produced significantly more cobs only than at control and in 2006, 2007 and combined seasons on cob diameter where $2 \mathrm{t}$ poultry manure ha ${ }^{-1}$ produced significantly wider cobs only than the control. Significant $(P<0.05)$ interaction was observed between intra-row spacing and poultry manure on cob diameter in 2007 rainy season, where application of 2 $\mathrm{t}$ poultry manure $\mathrm{ha}^{-1}$ produced significantly wider cobs (Table 4).

Table 3 shows the effects of intra-row spacing; nitrogen and poultry manure on cob length, 100 -grain weight and grain yield in all years and combined. Intra-row spacing had no significant effect on cob length at all the years and combined. Intrarow spacing significantly increase 100 -grain weight in 2006, 2007 and combined. 
In 2006; 2007 and combined 30cm intra-row spacing produced significantly heavier 100 -grain weight than $20 \mathrm{~cm}$. Intra-row spacing of $30 \mathrm{~cm}$ in 2006 and 2008 produced significantly lower grain yield than 20 and $25 \mathrm{~cm}$ which were statistically at par. In 2007, the highest grain yield was obtained at $25 \mathrm{~cm}$ intra-row spacing followed by 20 and $30 \mathrm{~cm}$ which were statistically at par. When the three years data were combined, $25 \mathrm{~cm}$ had the highest grain yield than $30 \mathrm{~cm}$ which produced significantly similar grain yield with $20 \mathrm{~cm}$.

Application of $120 \mathrm{~kg} \mathrm{~N}^{-1}$ produced significantly longer cobs, heavier 100-grain weight and higher grain yield in 2006, 2007 and combined seasons only than at $40 \mathrm{~kg} \mathrm{~N} \mathrm{ha}^{-1}$. Increase application of poultry manure from 0 to $4 \mathrm{t} \mathrm{ha}^{-1}$ gave statistically longer cobs in 2006 and 2007; further increase to $6 \mathrm{t} \mathrm{ha}^{-1}$ and beyond resulted in similar cob length but longer than at control. In 2008 and combined, there was no significant difference among the poultry manure rates. Similar trend was observed on grain yield in 2006 and combined on poultry manure application. Significant interactions between nitrogen and poultry manure was observed on 100-grain weight in 2006 and grain yield in2007; between intra-row spacing and poultry manure on grain yield in 2007; between intra-row spacing and nitrogen on 100-grain weight in 2006 and grain yield in 2007 (Tables 5 and 6).

\section{Regression Analysis}

The regression analysis of grain yield against nitrogen rates gave the following optimum yields 4868.94 at $107 \mathrm{~kg} \mathrm{~N} \mathrm{ha}^{-1}$ in 2006, $1702.86 \mathrm{~kg} \mathrm{ha}^{-1}$ at $94 \mathrm{~kg} \mathrm{~N} \mathrm{ha}^{-1}$ in 2007, $2039.7 \mathrm{~kg} \mathrm{ha}^{-1}$ at $72 \mathrm{~kg} \mathrm{~N} \mathrm{ha}^{-1}$ in 2008 and $2285.3 \mathrm{~kg} \mathrm{ha}^{-1}$ at $82 \mathrm{~kg} \mathrm{~N} \mathrm{ha}^{-1}$ in the combined (Fig 1). Similarly, when grain yield was regressed against poultry manure rates the optimum yields was 4298.00 $\mathrm{kg} \mathrm{ha}^{-1}$ at $3.35 \mathrm{t} \mathrm{ha}^{-1}, 1851.41 \mathrm{~kg} \mathrm{ha}^{-1}$ at $3.81 \mathrm{t} \mathrm{ha}^{-1}$, $2096.57 \mathrm{~kg} \mathrm{ha}^{-1}$ at $2.55 \mathrm{t} \mathrm{ha}^{-1}$ and $2260.12 \mathrm{~kg} \mathrm{ha}^{-1}$ at $1.91 \mathrm{t} \mathrm{ha}^{-1}$ for $2006,2007,2008$ and combined respectively (Fig 2 ).

Table 1: Chemical properties of soils at the experimental sites and poultry manure during 2006, 2007 and 2008 rainy seasons at Samaru.

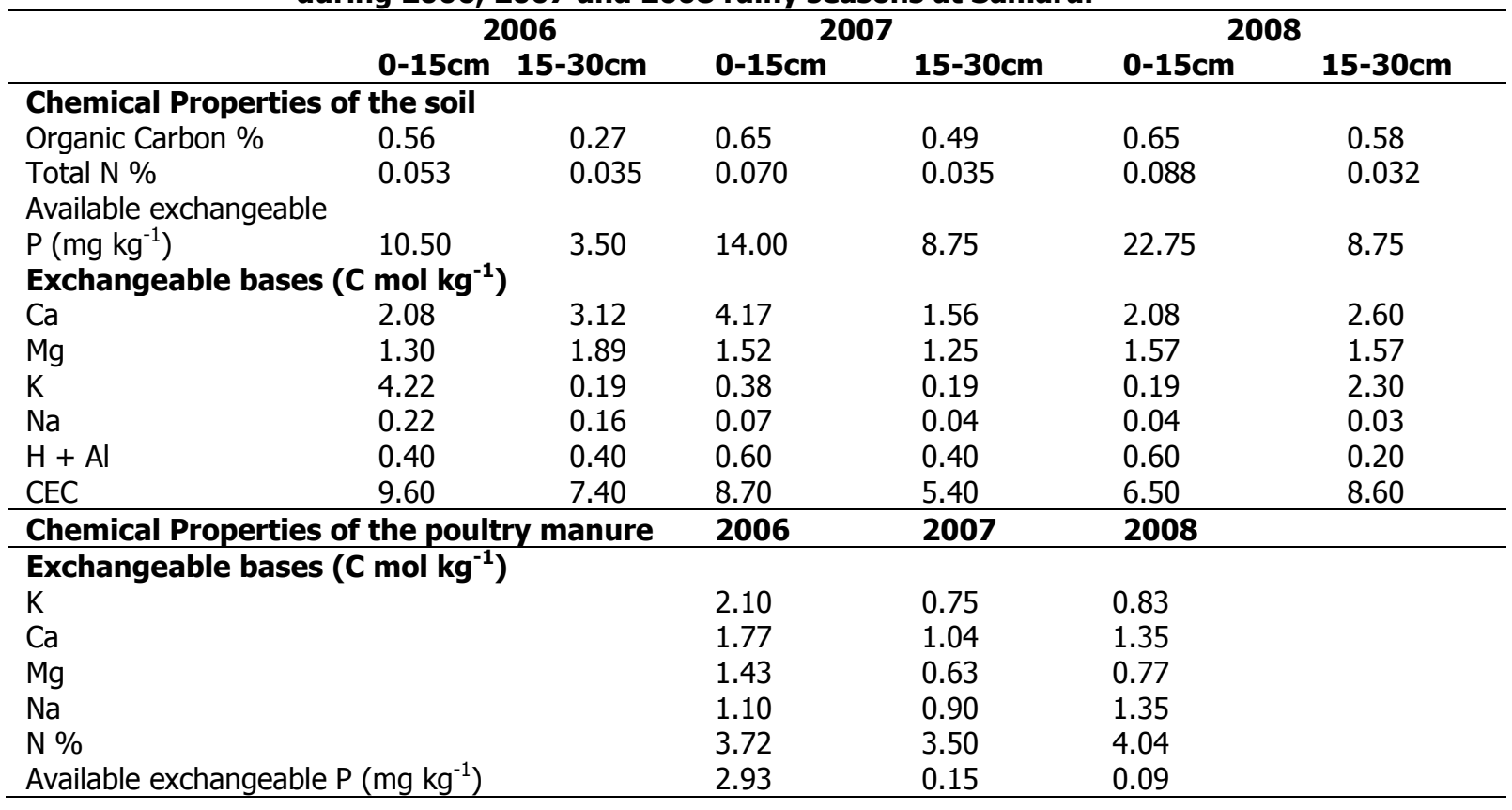


Bajopas Volume 5 Number 1 June, 2012

Table 2: Effects of intra-row spacing, nitrogen and poultry manure rates on number of cob plant ${ }^{-1}$, number of rows cob ${ }^{-1}$ and cob diameter $(\mathrm{cm})$ of extra early maize (Zea mays $\quad$ L.) during 2006, 2007 and 2008 rainy seasons at Samaru, Zaria.

\begin{tabular}{|c|c|c|c|c|c|c|c|c|c|c|c|c|}
\hline \multirow[b]{2}{*}{ Treatments } & \multicolumn{4}{|c|}{ Number of cob plant ${ }^{-1}$} & \multicolumn{4}{|c|}{ Number of rows $\mathrm{cob}^{-1}$} & \multicolumn{4}{|c|}{ Cob diameter $(\mathrm{cm})$} \\
\hline & 2006 & 2007 & 2008 & Combined & 2006 & 2007 & 2008 & Combined & 2006 & 2007 & 2008 & Combined \\
\hline \multicolumn{13}{|c|}{ Intra-row spacing (I) cm } \\
\hline 20 & 1.06 & 1.00 & 1.00 & $1.02 b$ & 14.36 & $13.09 \mathrm{~b}$ & $13.81 b$ & $13.39 \mathrm{~b}$ & $4.12 b$ & $5.85 b$ & 6.66 & $5.45 b$ \\
\hline 25 & 1.11 & 1.08 & 1.00 & $1.07 a b$ & 13.97 & 13.47ab & 14.03ab & 14.09a & $4.34 a$ & 6.07ab & 6.72 & 5.53ab \\
\hline 30 & 1.14 & 1.14 & 1.03 & $1.01 \mathrm{a}$ & 14.28 & $14.03 a$ & $14.53 a$ & $14.14 a$ & $4.39 a$ & $6.21 a$ & 6.81 & $5.76 a$ \\
\hline SE \pm & 0.053 & 0.048 & 0.016 & 0.024 & 0.181 & 0.334 & 0.197 & 0.148 & 0.045 & 0.110 & 0.192 & 0.078 \\
\hline \multicolumn{13}{|c|}{ Nitrogen $\mathrm{kg} \mathrm{ha}^{-1}(\mathrm{~N})$} \\
\hline 40 & 1.08 & 1.08 & 1.00 & 1.06 & 14.33 & 13.11 & 14.47 & 13.97 & $4.11 \mathrm{~b}$ & $5.98 b$ & $6.38 \mathrm{~b}$ & $5.45 b$ \\
\hline 80 & 1.11 & 1.06 & 1.00 & 1.06 & 14.14 & 13.75 & 13.94 & 13.94 & $4.38 a$ & $6.12 \mathrm{a}$ & $6.78 a$ & $5.76 a$ \\
\hline 120 & 1.11 & 1.08 & 1.03 & 1.08 & 14.14 & 13.64 & 13.94 & 13.91 & 4.37a & $6.03 a$ & $6.72 \mathrm{ab}$ & 5.70ab \\
\hline SE \pm & 0.053 & 0.039 & 0.016 & 0.023 & 0.158 & 0.374 & 0.263 & 0.137 & 0.0645 & 0.139 & 0.214 & 0.092 \\
\hline \multicolumn{13}{|c|}{ Poultry manure t ha ${ }^{-1}$ (PM) } \\
\hline 0 & 1.04 & 1.00 & 1.00 & $1.00 \mathrm{~b}$ & 14.00 & 14.19 & 14.19 & 14.12 & $4.22 b$ & $5.69 b$ & 6.43 & $5.31 b$ \\
\hline 2 & 1.11 & 1.15 & 1.00 & $1.05 \mathrm{ab}$ & 14.41 & 14.15 & 13.78 & 14.11 & $4.36 a$ & $6.12 \mathrm{a}$ & 6.67 & $5.66 \mathrm{a}$ \\
\hline 4 & 1.11 & 1.04 & 1.00 & $1.09 a$ & 13.96 & 13.78 & 14.15 & 13.96 & $4.38 a$ & $6.13 a$ & 6.97 & $5.75 a$ \\
\hline 6 & 1.15 & 1.11 & 1.04 & $1.10 \mathrm{a}$ & 14.44 & 11.89 & 14.37 & 13.75 & $4.45 a$ & $6 . .23 a$ & 6.84 & $5.83 a$ \\
\hline $\mathrm{SE} \pm$ & 0.061 & 0.045 & 0.019 & 0.026 & 0.182 & 0.432 & 0.303 & 0.155 & 0.0745 & 0.160 & 0.247 & 0.103 \\
\hline \multicolumn{13}{|l|}{ Interaction } \\
\hline NxPM & NS & NS & NS & NS & NS & NS & NS & NS & NS & NS & NS & NS \\
\hline IxPM & NS & NS & NS & NS & NS & NS & NS & NS & NS & $*$ & NS & NS \\
\hline IxN & NS & NS & NS & NS & NS & NS & NS & NS & NS & NS & NS & NS \\
\hline NxIxPM & NS & NS & NS & NS & NS & NS & NS & NS & NS & NS & NS & NS \\
\hline
\end{tabular}

Any two means not sharing a letter differ significantly from each other at $5 \%$ probability level. NS= not significant, $*=$ significant at $P<0.05$ 
Bajopas Volume 5 Number 1 June, 2012

Table 3: Effects of intra-row spacing, nitrogen and poultry manure rates on cob length $(\mathrm{cm}), \mathbf{1 0 0 - g r a i n ~ w e i g h t ~}(\mathrm{g})$ and grain yield $\left(\mathrm{kg} \mathrm{ha}^{-1}\right.$ ) of extra early maize (Zea mays L.) during 2006- 2008 and combined rainy seasons at Samaru, Zaria.

\begin{tabular}{|c|c|c|c|c|c|c|c|c|c|c|c|c|}
\hline \multirow[b]{2}{*}{ Treatments } & \multicolumn{4}{|c|}{ Cob length $(\mathrm{cm})$} & \multicolumn{4}{|c|}{ 100-grain weight (g) } & \multicolumn{4}{|c|}{ Grain yield $\left(\mathrm{kg} \mathrm{ha}^{-1}\right)$} \\
\hline & 2006 & 2007 & 2008 & Combined & 2006 & 2007 & 2008 & Combined & 2006 & 2007 & 2008 & Combined \\
\hline \multicolumn{13}{|c|}{ Intra-row spacing (I) cm } \\
\hline 20 & 14.01 & 11.30 & 13.19 & 12.84 & $19.63 b$ & $17.54 b$ & 20.97 & $19.64 b$ & $3121 a$ & $1493 b$ & $1923 a$ & $2189 a b$ \\
\hline 25 & 14.03 & 10.94 & 13.53 & 12.83 & $20.60 \mathrm{ab}$ & $19.05 a b$ & 20.44 & $20.33 a b$ & $3294 a$ & $1679 a$ & $2349 a$ & $2316 a$ \\
\hline 30 & 13.81 & 11.75 & 13.12 & 12.90 & $21.45 a$ & $19.41 a$ & 21.51 & $20.51 a$ & $2572 b$ & $1426 b$ & $1563 b$ & $1968 b$ \\
\hline $\mathrm{SE} \pm$ & 0.206 & 0.336 & 0.541 & 0.226 & 0.385 & 0.630 & 0.357 & 0.266 & 188.9 & 57.8 & 123.1 & 81.3 \\
\hline \multicolumn{13}{|c|}{ Nitrogen kg ha-1 (N) } \\
\hline 40 & $13.06 \mathrm{~b}$ & $10.56 \mathrm{~b}$ & 13.31 & $12.49 b$ & $20.26 b$ & $17.64 b$ & 20.96 & $20.01 b$ & $2598 b$ & $1269 b$ & 1990 & $1838 b$ \\
\hline 80 & 13.91ab & $11.64 a$ & 13.09 & $12.88 a b$ & $21.02 \mathrm{ab}$ & $19.96 a$ & 21.09 & $20.45 a b$ & $3149 a$ & $1699 a$ & 2101 & $2103 a b$ \\
\hline 120 & $14.34 a$ & $11.74 a$ & 13.46 & $13.20 \mathrm{a}$ & $21.60 a$ & $19.88 \mathrm{a}$ & 20.88 & $21.69 a$ & $3377 a$ & $1633 a$ & 1955 & $2317 a$ \\
\hline SE \pm & 0.237 & 0.429 & 0.534 & 0.230 & 0.469 & 0.671 & 0.304 & 0.299 & 192.2 & 193.2 & 144.7 & 128.1 \\
\hline \multicolumn{13}{|c|}{ Poultry manure t ha-1 (PM) } \\
\hline 0 & $13.26 b$ & $10.46 b$ & 13.54 & 12.73 & $20.26 b$ & 19.13 & 19.63.b & $19.37 b$ & $2756 b$ & $973 c$ & $1568 b$ & $1836 b$ \\
\hline 2 & 13.89ab & 11.39ab & 12.65 & 12.73 & $20.49 b$ & 19.47 & $21.23 a$ & $20.40 a$ & 2969ab & $1643 b$ & $1669 b$ & $2103 a b$ \\
\hline 4 & $14.43 a$ & $11.65 a$ & 12.62 & 12.96 & $22.11 a$ & 19.42 & $21.15 a$ & $20.89 a$ & $3532 a$ & $1848 a$ & $2037 a b$ & $2285 a$ \\
\hline 6 & $14.23 a$ & $11.82 \mathrm{a}$ & 14.34 & 13.01 & $21.98 a$ & 18.88 & $20.89 a$ & $20.24 a$ & $3287 a b$ & $1672 a b$ & $2143 a$ & $2358 a$ \\
\hline $\begin{array}{l}\mathrm{SE} \pm \\
\text { Interaction }\end{array}$ & 0.273 & 0.495 & 0.616 & 0.266 & 0.542 & 0.775 & 0.351 & 0.346 & 221.9 & 223.0 & 167.1 & 147.9 \\
\hline NxPM & NS & NS & NS & NS & $*$ & NS & NS & NS & NS & $*$ & NS & NS \\
\hline IxPM & NS & NS & NS & NS & NS & NS & NS & NS & NS & $*$ & NS & NS \\
\hline $\mathrm{IxN}$ & NS & NS & NS & NS & $*$ & NS & NS & NS & NS & $*$ & NS & NS \\
\hline NxIxPM & NS & NS & NS & NS & NS & NS & NS & NS & NS & NS & NS & NS \\
\hline
\end{tabular}

Any two means not sharing a letter differ significantly from each other at $5 \%$ probability level. NS $=$ not significant, $*=$ significant at

$P<0.05$ 
Bajopas Volume 5 Number 1 June, 2012

Table 4: Interaction between Intra-row spacings and poultry manure on cob diameter in 2007 rainy season

\begin{tabular}{llcl}
\hline & \multicolumn{3}{c}{ Intra-row spacing (cm) } \\
\cline { 2 - 4 } Treatment & $\mathbf{2 0}$ & $\mathbf{2 5}$ & $\mathbf{3 0}$ \\
\hline Poultry manure t ha $\mathbf{~}^{\mathbf{1}}$ & & & \\
0 & $5.93 \mathrm{~d}$ & $5.01 \mathrm{~d}$ & $6.22 \mathrm{a}-\mathrm{c}$ \\
2 & $5.74 \mathrm{a}$ & $6.53 \mathrm{a}$ & $6.41 \mathrm{ab}$ \\
4 & $6.29 \mathrm{ab}$ & $6.02 \mathrm{a}-\mathrm{c}$ & $6.08 \mathrm{a}-\mathrm{c}$ \\
6 & $6.30 \mathrm{ab}$ & $5.83 \mathrm{bc}$ & $6.11 \mathrm{a}-\mathrm{c}$ \\
$\mathrm{SE} \pm \mathrm{N}$ & 0.221 & &
\end{tabular}

Any two means not sharing a letter differ significantly from each other at 5\% probability level.

Table 5: Interaction between nitrogen and poultry manure on 100-grain weight in 2006 rainy season

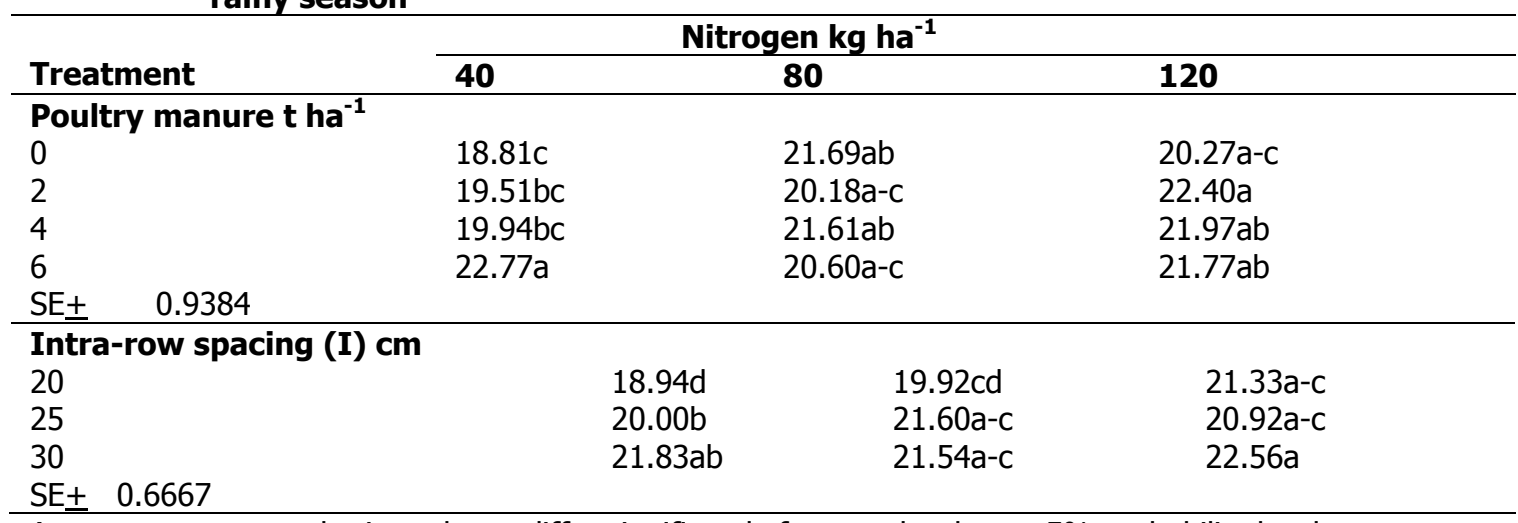

Any two means not sharing a letter differ significantly from each other at $5 \%$ probability level.

Table 6: Interaction of nitrogen $x$ intra-row spacing, nitrogen $x$ poultry manure and intra -row spacing $x$ poultry manure on grain yield in 2007 rainy season

\begin{tabular}{llcc}
\hline $\mathbf{6 a}$ & \multicolumn{3}{c}{ Nitrogen $\mathbf{~ k g ~ h a} \mathbf{~}^{\mathbf{- 1}}$} \\
\cline { 2 - 4 } Treatment & $\mathbf{4 0}$ & $\mathbf{8 0}$ & $\mathbf{1 2 0}$ \\
\hline Intra-row spacing (I) cm & $1324.12 \mathrm{~cd}$ & $1580.83 \mathrm{bc}$ & $1584.92 \mathrm{bc}$ \\
20 & $1065.33 \mathrm{~d}$ & $1912.90 \mathrm{a}$ & $1607.00 \mathrm{bc}$ \\
25 & $1418.04 \mathrm{c}$ & $1605.97 \mathrm{bc}$ & $1708.15 \mathrm{ab}$ \\
30 & & & \\
$\mathrm{SE} \pm 100.16$ & & & \\
\hline
\end{tabular}

\begin{tabular}{|c|c|c|c|}
\hline \multirow{2}{*}{$\begin{array}{l}\text { 6b } \\
\text { Treatment }\end{array}$} & \multicolumn{3}{|c|}{ Nitrogen $\left(\mathrm{kg} \mathrm{ha}^{-1}\right)$} \\
\hline & 40 & 80 & 120 \\
\hline \multicolumn{4}{|c|}{ Poultry manure t ha ${ }^{-1}$} \\
\hline 0 & $3146.2 a-c$ & $2334.7 c$ & $3240.9 a-c$ \\
\hline 2 & $3387.9 a-c$ & $4156.1 a$ & $2841.2 b c$ \\
\hline 4 & $3927.4 a b$ & 34.28.7a-c & $2787.4 c$ \\
\hline 6 & $3045.5 b c$ & $2677.6 c$ & $2657.8 c$ \\
\hline$S E \pm \quad 384.47$ & & & \\
\hline \multicolumn{4}{|c|}{ ow spacing (cm) } \\
\hline Treatment & 20 & 25 & 30 \\
\hline \multicolumn{4}{|c|}{ Poultry manure t ha ${ }^{-1}$} \\
\hline 0 & 958.83ef & 1059.70ef & $902.33 f$ \\
\hline 2 & 1977.30a & 1695.17a-c & $1870.37 a b$ \\
\hline 4 & $1408.50 \mathrm{~cd}$ & $1661.30 \mathrm{bc}$ & $1946.21 \mathrm{ab}$ \\
\hline 6 & $1641.87 \mathrm{bc}$ & 1288.24de & 1999.88a \\
\hline SE $\pm \quad 115.65$ & & & \\
\hline
\end{tabular}

Any two means not sharing a letter differ significantly from each other at 5\% probability level. 


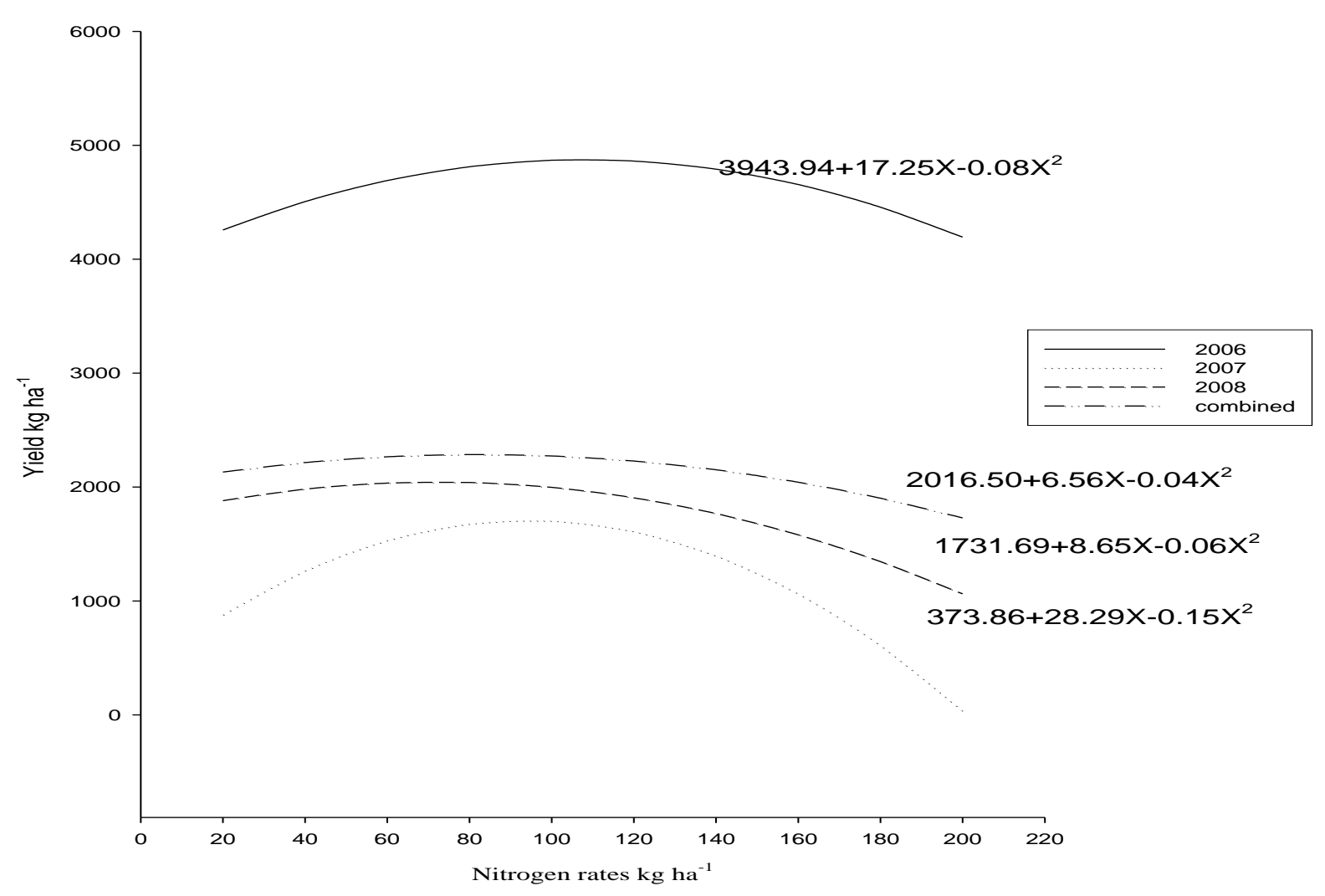

Fig 1: Regresion response of nitrogen rate against grain yield in 2006, 20072008 and combined

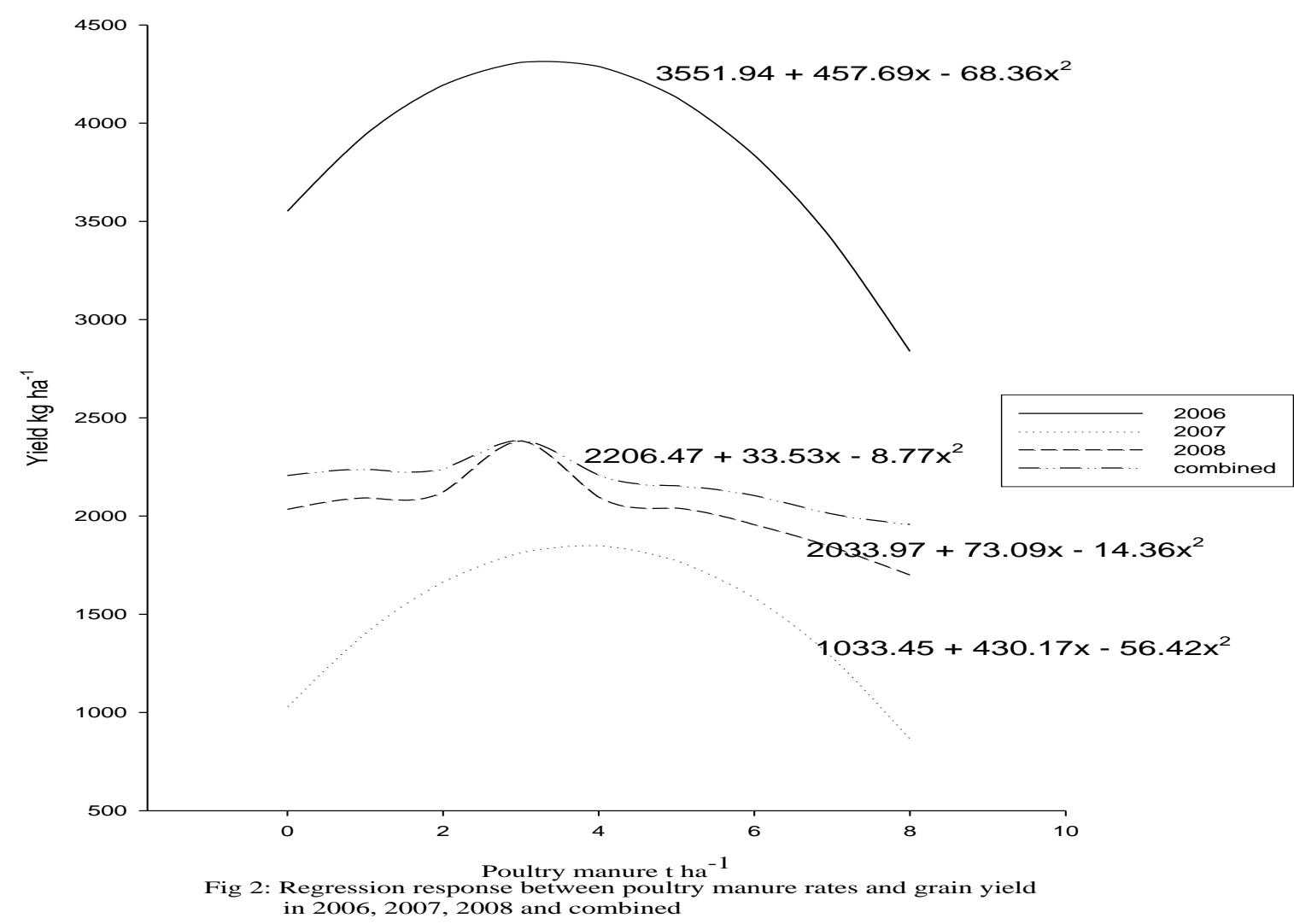


Appendix I: Samaru Meteorological Observation in 2006, 2007 and 2008 Rainy Seasons.

\begin{tabular}{|c|c|c|c|c|c|c|c|c|c|c|c|c|c|c|c|}
\hline \multirow{3}{*}{ Month } & \multicolumn{3}{|c|}{ Rainfall (mm) } & \multicolumn{6}{|c|}{ Temperature $\left({ }^{\circ} \mathrm{C}\right)$} & \multicolumn{6}{|c|}{ Relative Humidity (\%) } \\
\hline & \multirow[b]{2}{*}{2006} & \multirow[b]{2}{*}{2007} & \multirow[b]{2}{*}{2008} & \multicolumn{3}{|c|}{ Minimum } & \multicolumn{3}{|c|}{ Maximum } & \multicolumn{3}{|c|}{$10.00 \mathrm{H}$} & \multicolumn{3}{|c|}{$16.00 \mathrm{H}$} \\
\hline & & & & 2006 & 2007 & 2008 & 2006 & 2007 & 2008 & 2006 & 2007 & 2008 & 2006 & 2007 & 2008 \\
\hline January & - & - & - & 15.7 & 12.9 & 13.6 & 34.8 & 28.8 & 29.0 & 15.5 & 18.4 & 19.7 & 14.4 & 12.4 & 15.1 \\
\hline February & - & - & - & 18.3 & 16.3 & 15.7 & 37.2 & 34.9 & 32.0 & 20.4 & 13.0 & 12.7 & 14.5 & 9.8 & 9.4 \\
\hline March & - & - & - & 28.3 & 19.0 & 19.9 & 58.7 & 37.1 & 38.6 & 27.9 & 19.2 & 19.5 & 21.3 & 9.2 & 13.8 \\
\hline April & 1.6 & - & 72.6 & 21.4 & 22.8 & 29.1 & 38.5 & 37.8 & 57.2 & 25.0 & 48.4 & 34.0 & 16.3 & 30.2 & 23.9 \\
\hline May & 121.9 & 194.8 & 95.0 & 21.6 & 21.8 & 21.9 & 33.6 & 34.0 & 35.0 & 71.0 & 68.0 & 63.0 & 49.5 & 53.6 & 46.2 \\
\hline June & 126.5 & 109.0 & 111.7 & 21.1 & 20.4 & 20.9 & 32.2 & 31.2 & 33.1 & 72.3 & 76.9 & 72.3 & 56.8 & 62.7 & 55.1 \\
\hline July & 232.2 & 228.6 & 201.3 & 20.8 & 20.8 & 20.0 & 30.8 & 29.8 & 30.5 & 78.8 & 79.6 & 79.6 & 66.6 & 66.9 & 68.0 \\
\hline August & 215.5 & 328.0 & 352.6 & 20.4 & 21.3 & 19.5 & 29.9 & 28.8 & 29.7 & 81.5 & 77.0 & 82.0 & 69.1 & 74.3 & 73.2 \\
\hline September & 281.5 & 31.9 & 217.5 & 20.2 & 20.2 & 25.5 & 30.9 & 31.5 & 31.4 & 80.1 & 76.2 & 77.1 & 70.5 & 59.1 & 66.0 \\
\hline October & 28.5 & 8.3 & 89.0 & 20.7 & 18.2 & 18.2 & 32.3 & 34.9 & 33.2 & 68.0 & 59.0 & 58.8 & 55.5 & 38.5 & 51.8 \\
\hline November & - & - & - & 13.6 & 15.2 & 12.8 & 31.1 & 35.3 & 33.8 & 22.2 & 22.8 & 21.2 & 22.3 & 18.0 & 23.1 \\
\hline December & - & - & - & 12.5 & 13.5 & 14.6 & 30.6 & 32.5 & 32.1 & 18.2 & 17.6 & 20.8 & 14.5 & 16.1 & 17.8 \\
\hline Total & 1007.9 & 900.6 & 1140.0 & 234.6 & 222.4 & 231.7 & 420.6 & 397.0 & 415.6 & 580.9 & 576.1 & 560.7 & 471.3 & 450.8 & 463.4 \\
\hline Mean & 84.0 & 75.1 & 95.0 & 19.6 & 18.5 & 19.3 & 35.1 & 33.1 & 34.6 & 48.4 & 48.0 & 46.7 & 39.9 & 37.6 & 38.6 \\
\hline
\end{tabular}

Source: Meteorological Unit, Institute for Agricultural Research Ahmadu Bello University, Zaria, Nigeria

\section{Discussion}

The higher yield and yield components observed in 2006 could be attributed to better weather condition compared to the other years. The minimum day temperature during the growing season $\left(18.6^{\circ} \mathrm{C}\right)$ in 2006 was lower especially when compared with the 2007 and $2008\left(19.6\right.$ and $\left.19.3^{\circ} \mathrm{C}\right)$ growing seasons. The mean temperature and relative humidity recorded at the time of pollination and fertilization in 2006 2007, 2008 were $19.6{ }^{\circ} \mathrm{C}$ and $48.4 \% ; 18.5^{\circ} \mathrm{C}$ and $48 \% ; 19.3{ }^{\circ} \mathrm{C}$ and $46.7 \%$, respectively (Appendix I). It is known that higher air temperature adversely affected crop productivity (Hussaini et al., 2001). The mean rainfall in 2006 was $84 \mathrm{~mm}$ and was evenly distributed from July to September and this period corresponds with flowering and grain filling stages. However in 2007, there was early cessation of rainfall at the end of September and was not evenly distributed (Appendix I). In 2008 rainy season there was more rain and it was not evenly distributed (Appendix I) and water logging at the center of the field was observed. This could cause the leaching of the soil nutrients down the soil profile beyond the root zone of the crop or the nutrients washed away, consequently causing reduction in yield.
Yield and yield components studied responded significantly $(P<0.05)$ to variation in intra-row spacing. Number of cobs plant ${ }^{-1}$, number of rows $\operatorname{cob}^{-1}$, cob diameter were all significantly decreased when intra-row spacing decreased from 30 to $20 \mathrm{~cm}$. This resulted in limited supplies of carbon and nitrogen and consequent increase in barrenness and decrease in grain number plant ${ }^{-1}$ as result of intense intra-plant competition for light, soil nutrient and soil water. Maize intra-row spacing for maximum economic yield varies from 18 to $45 \mathrm{~cm}$ depending on planting date, water availability, soil fertility and maturity (Sangoi, 2001). The increase in yield might be due to better light utilization by the closely spaced plants, hence higher dry matter for grain filling. This result was in agreement with the findings of Okan et al. (2004) who obtained highest yield from lowest intra-row spacing $(20 \mathrm{~cm})$.

Nitrogen fertilization significantly increased extra early maize development. The trend was manifested in all seasons. Yield component such as cob diameter, 100-grain weight and grain yield ha-1 were all optimized at $80 \mathrm{~kg} \mathrm{~N} \mathrm{ha}^{-1}$. This could be due to the fact that excessive nitrogen reduce grain yield but enhanced plant growth. 
This finding corroborated those of Hussaini et al. (2001), Mani (2004), Sharifai et al. (2008) and Namakka et al. (2009). 100-grain weight was significantly increased with nitrogen application up to $80 \mathrm{~kg} \mathrm{ha}^{-1}$. This is because an increase in nitrogen application positively enhance the chlorophyll content in plant thereby improving photosynthetic activities that promotes assimilate production and this will result in the increase in final yield. Nitrogen fertilization significantly $(P<0.005)$ increased maize grain yield with each increase in nitrogen between 40 to $80 \mathrm{~kg}$ $\mathrm{ha}^{-1}$. Hussaini, et al. (2001) reported similar response where they attributed this significant increase in yield to favourable effect of nitrogen on cob length and cob diameter, which all have direct bearing on the final grain yield. The increase in yield could be as a result of good dry matter production for grain filling as a result of higher number of leaves.

It was observed that poultry manure application significantly increased most of the yield components compared with plots that received no poultry manure. Yield and yield components of maize such as cob diameter and 100-grain weight were all highest at $4 \mathrm{t}$ poultry manure $\mathrm{ha}^{-1}$ in 2006, 2007 and combined means. This could be attributed to the ability of poultry manure to supply essential nutrients and micronutrients for crop production. They are also valuable sources of soil organic matter. Organic matter improves soil structure; increases water holding capacity of soil and are source of slow-release nutrient. Soil organic matter also contributes to greater efficiency of fertilizer use.

The significant response of 100 -grain weight and grain yield to applied nitrogen and poultry manure signified the importance of $\mathrm{N}$ in the development of maize. The increase in these parameters could be due to synergy effects of nitrogen and poultry manure. The interactions ascertain the affirmation that the combined application of both organic and inorganic

\section{REFERENCES}

Alofe, C.O., Kim, S.K., Efron, Y., Fajemisin, J.M., Khadr, F.H. and Kang, B.T (1988). Effect of nitrogen and plant density on grain performance of hybrid maize in the forest and derived savanna zone of Nigeria. National Conference on maize, Ile-Ife Abstract 17pp

Badu-Apraku, B., Menkir, A., Fakorede, M.A.B., Fonten Lum, A. and K., Obeng-Antwi (2006) Multivariate analyses of the genetic diversity of forty-seven Striga resistant tropical early maturing maize inbred lines. Maydica 51: 551-559

Barr, A.J. and Goodnight, J.H. (1972). A user guide to the statistical analysis system. Department of statistics. North Cardenas State University pp107-114

Boateng, S. A, Zickermann, J. and Kornahrens, M. (2006). Poultry Manure Effect on Growth and manure is essential for crop yield. Boateng et al. (2006) reported similar result where interaction between $\mathrm{N}$ and Poultry manure increases the yield of maize crop and improve the soil structures and chemical nutrients of the soil.

The significant interaction between intra-row spacing and poultry manure on cob diameter, 100 grain weight and grain yield showed the importance of poultry manure on yield and yield components of maize crop. Poultry manure increases both soil physical and chemical properties. The yield increase with poultry manure application at $2 \mathrm{t} \mathrm{ha}^{-1}$ at closer spacing might be due to availability of nutrients for crop use.

The significant interaction between intra-row spacing and nitrogen on grain yield showed the importance of nitrogen on yield of maize crop. The yield increase with higher nitrogen rates at closer spacing might be due to improved rooting depth, leaf area expansion and thus the efficiency in the use of solar radiation which indirectly increases dry matter production for grain filling, therefore increases yield. Tenaw (2000) reported similar trend where nitrogen $\mathrm{x}$ intra-row spacing gave yield component and yield at the lowest intra-row spacing of $18 \mathrm{~cm} \times 60 \mathrm{~kg} \mathrm{~N} \mathrm{ha}^{-1}$.

From the results of the regression analysis it was observed that optimum yield was a combination of 20 $\mathrm{cm}$ intra-row spacing, $82 \mathrm{~kg} \mathrm{~N} \mathrm{ha}^{-1}$ and $1.91 \mathrm{t}$ poultry manure ha ${ }^{-1}$ in combined.

Based on the results obtained it can be concluded that optimum grain yield of extra early maize was obtained at $25 \mathrm{~cm}$ intra-row spacing, $82 \mathrm{~kg} \mathrm{~N} \mathrm{ha}^{-1}$ and $1.91 \mathrm{t}$ poultry manure ha ${ }^{-1}$.

\section{Acknowledgement}

We acknowledge the support of Cereals Research Programme of the Institute for Agricultural Research (I.A.R.), University Board of Research (U.B.R.) and technical assistance by Mr. I. M. Aboh.

Yield of maize. Crop Science Journal, 8 (3): 273-282

Duncan, D.B. (1955). Multiple range and multiple Ftest. Biometrics 11: 1-42

Elemo, K.A (1997). Extra-early maize varieties as affected by the rate and time of $\mathrm{N}$ fertilization. Production of reginal maize workshop IITA-Cotonou, Benin Republic: 419-425.

FAO (2010). Food and Agriculture Organisation Statistics, FAOSTAT. www.fao.org/faostat

Garg, J.S. and Bansal, M.G. (1972). Optimum dose of nitrogen for high yielding dwarf wheat. Fertilizer News 17(10): 53-55

Hussaini, M.A., Ogunlela, V.B., Ramalan, A.A. and Falaki, A.M. (2001). Growth and development of maize (Zea mays L.) in response to different levels of nitrogen, phosphorus and irrigation. Crop Research 22 (2): 141-149 
Kamara, A.Y. and Sanginga, N (2001). Balance nutrient management for intensified maizebased systems in the Northern Guinea Savanna of West Africa. In: Proceeding of the National Quality Protein Maize Production Workshop $4^{\text {th }}-5^{\text {th }}$ September 2001 at Institute for Agricultural Research Comperence Hall A.B.U., Zaria pp 17-24.

Luis, S (2001). Understanding plant density effects on maize growth and development: An important issue to maximize grain yield. Ciencia Rura/ 3(1): 1-17

Mani, H (2004). Growth and yield performance of two popcorn (Zea mays everta) varieties to rates of NPK fertilizer under different irrigation intervals. Unpublished Ph.D dissertation, submitted to postgraduate school, Ahmadu Bello University, Zaria. Pp 167

Manyong, V.M., Smith, J., Webe, G.K., Jagtap, S.S and Oyewole, B (1996). Macro-characterization of agricultural systems in West Africa: An Over view Research and Crop Management Research Monograph No. 21. International Institute of Tropical Agriculture (IITA), Ibadan, Nigeria.

Namakka, A., I.U. Abubakar, I.A. Sadiq, A.I. Sharifai, A.H. Hassan and Y. Hussaini (2009). Effects of varying sowing date and nitrogen level on growth of two extra-early maize (Zea mays L.) varieties in Sudan Savanna of Nigeria. Journal of Agricultural Research and Policies 1(1): $30-34$
Okan, S., Huseyin, G., Omer, K and Mehmet, K (2004). The effect of intra-row spacing on grain yield and some agronomic characteristics of maize (Zea mays L) hybrids. Asian Journal of Plant Sciences 3(4): 429-432

Olson, R.A and D.H. Sanders (1988). Crop production in corn and corn improvement Madison W.I. U.S.A: 686

Reddy, R.S., Ahmad, M.K., and Cheri, A.V. (1975). Polynomial response of $\mathrm{CSH}-1$ sorghum to varying level of nitrogen and phosphorus. Journal of Agricultural Research. APAU IV (3 and 4): 81-83

SAS Institute Inc (1990). SAS/STAT User's guide, version $6^{\prime} 4^{\text {th }}$ edn. SAS Institute Inc. Cary, NC, USA

Sangoi, L (2001). Understanding plant density effect on maize growth and development: an important issue to maximize grain yield. Ciencia Rural. 31: 159-168

Sharifai, A.I., Mahmud, M., Mahadi, M.A., Kura, H.N and Namakka, A (2008). Effect of different level of NPK compound fertilizer on growth parameters of extra-early maize (Zea mays L.) varieties. Journal of Agricultural Research and Policies 3 (2): 65-69

Tenaw, W. (2000). Effects of plant population and nitrogen doses on fresh ear yield components of sweet corn (Zea mays saccharata Sturt) Grown under Bursa conditions. Turkish Journal of Agriculture and Forestry 24: 341347 